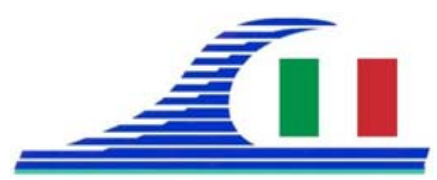

Conférence Méditerranéenne Côtière et Maritime EDITION 3, FERRARA, ITALIA (2015)

Coastal and Maritime Mediterranean Conference

Disponible en ligne - http://www.paralia.fr - Available online

\title{
Modélisation numérique de l'aléa de submersion appliquée à l'élaboration des plans d'évacuation et à la gestion de crise, exemple de la commune de Leucate
}

\author{
Alexandre NICOLAE LERMA ${ }^{1}$, François PARIS ${ }^{1}$, Sylvain ELINEAU ${ }^{1,3}$, \\ Yann BALOUIN ${ }^{2}$, Sophie LECACHEUX ${ }^{1}$, Paul DURAND ${ }^{3}$, Brice ANSELME ${ }^{4}$, \\ Esméralda LONGEPE ${ }^{4}$, Stéphanie DEFOSSEZ ${ }^{5}$, Lydie GOELDNER GIANELLA $^{3}$
}

1. BRGM, Direction Risques et Prévention, Unité Risques Côtiers et Changement Climatique, Orléans, 3 avenue Claude Guillemin, BP36009 - 45060 Orléans, France. a.nicolaelerma@brgm.fr,f.paris@brgm.fr,s.lecacheux@brgm.fr

2. BRGM, Direction régionale Languedoc-Roussillon, 1039 rue de Pinville, 34000 Montpellier.Y.balouin@brgm.fr

3. Laboratoire de Géographie Physique, UMR 8591 / Université Paris, 1 Place Aristide Briand 92195 Meudon Cedex.elineau.sylvain@gmail.com; Paul.Durand@univparis1.fr ; Lydie.Goeldner-Gianella@univ-paris1.fr

4. UMR 8586 PRODIG / Université Paris 1, Brice.Anselme@univ-paris1.fr

5. UMR GRED / Université Montpellier 3, stephanie.defossez@univ-montp3.fr

\section{Résumé :}

Le Projet CRISSIS vise à développer une approche intégrée et opérationnelle du risque de submersion marine sur une commune où se concentrent de forts enjeux urbains et touristiques et où l'aléa de submersion marine, important actuellement, tend à augmenter compte tenu de l'élévation progressive du niveau marin. Le travail présenté consiste en une modélisation fine des processus marins et lagunaires à l'origine des submersions sur la commune de Leucate (Aude). D’une part, les outils de modélisation mis en place permettent d'intégrer l'ensemble des contributions à l'origine des surélévations des niveaux d'eau lors des tempêtes et des modalités de la submersion (débordement, franchissement de paquet de mer, rupture d'ouvrage). D’autre part, les méthodes de traitement de l'information topo-bathymétrique et l'intégration des spécificités des environnements urbains (bâtiments, murets, ouvrages hydraulique, rugosité du sol) permettent de tenir compte des particularités des écoulements à terre. Cette approche réaliste validée par la reproduction d'évènements historiques, permet non seulement d'identifier les secteurs à risques, mais également d'évaluer les temporalités et les délais d'intervention possibles entre le début de l'évènement et la mise hors d'usage des axes de communication ou de bâtiments stratégiques. Les résultats visent à être utilisés lors d'exercices de crise et être confrontés aux représentations et à la perception du risque par la population

Mots-clés : Submersion marine, Lido, Lagune, Méditerranée, MARS-SWAN, SWASH, Projet CRISSIS. 
Côtes méditerranéennes menacées :

Risques et défis dans le contexte du changement climatique

\section{Introduction}

Depuis quelques années, les études traitant du risque submersion marine sur les côtes méditerranéennes se multiplient (BALOUIN \& BELON, 2011 ; BALOUIN et al., 2012 ; VINCHON et al., 2010 ; PEDREROS et al., 2011 ; ANSELME et al., 2011). Les méthodes mises en place contribuent à l'amélioration des connaissances du risque. Toutefois, ces approches restent majoritairement simples et sont parfois mal adaptées à une vision réaliste de l'aléa dans des configurations complexes telles que des milieux lagunaires et des lidos dont la topographie est généralement basse. Au sein de ces environnements où se concentrent de forts enjeux urbains, économiques et touristiques, des études complémentaires sont nécessaires pour permettre une meilleure acceptation du risque par la population et une amélioration de la gestion opérationnelle des risques et des crises.

La modélisation numérique, aujourd'hui couramment utilisée pour l'étude des aléas littoraux, doit être adaptée aux particularités d'exposition du site et aux phénomènes à l'origine des submersions : débordement, franchissement par paquets de mer, rupture de digues, etc. (GARCIN et al., 2012 ; LEROY et al., 2014). Les avancées récentes, tant du point de vue des développements techniques que des méthodes de pré et posttraitement, permettent la prise en compte à la fois des phénomènes qui concourent aux inondations (élévation du niveau marin moyen, action des vagues, apports fluviaux) et de leur chronologie (durée des submersions, rupture d’ouvrages de protection, concomitance ou non des maxima d'influences marine et fluviale). Il devient alors possible non seulement d'identifier les secteurs à risques, mais également d'évaluer les temporalités et les délais d'intervention possibles entre le début de l'évènement et la mise hors d'usage des axes de communication ou de bâtiments stratégiques.

L'approche proposée dans le cadre du programme CRISSIS, en partenariat avec la commune de Leucate (Languedoc-Roussillon), consiste dans un premier temps à réaliser une étude des évènements tempétueux passés pour caler et valider les résultats des modèles. Plusieurs scénarios (situation actuelle, en 2050 et en 2100) seront ensuite simulés et les résultats confrontés aux représentations et à la perception du risque par la population. Les informations sur les niveaux d'eau, les vitesses des flux et la chronologie des submersions seront par ailleurs utilisées lors d'un exercice de crise pour tester l’opérationnalité du Plan Communal de Sauvegarde adopté en 2013.

\section{Matériels et méthodes}

\subsection{Les forçages météo-marins}

\subsubsection{Niveaux d'eau}

Les niveaux d'eau utilisés sont issus des mesures marégraphiques de Sète et PortVendres (la commune de Leucate ne dispose pas de marégraphe). Une analyse 
statistique des valeurs de surcotes extrêmes a été réalisée pour ces 2 ports (CETMEF). Une étude précise des données sera effectuée pour chaque tempête historique. Les scénarios envisagés en terme de niveau marin seront construits à partir des observations et analyses de ces ports, et intégreront une concomitance entre la PHMA et le pic de surcote.

\subsubsection{Vagues}

La reproduction des évènements historiques et l'élaboration des scénarios reposent sur l'exploitation de données d'observation (bouée réseau CANDHIS, période 2007-2015), et de données issues des bases ANEMOC (source CETMEF, période 1979-2009) et GoLWA (Gulf of Lion Wave Atlas, source BRGM, période 1979-2009).

Tableau 1. Données de forçages disponibles pour le site d'étude, surcotes et hauteurs significatives des vagues en $m$.

\begin{tabular}{cccccc}
\hline $\begin{array}{c}\text { Période } \\
\text { de retour }\end{array}$ & $\begin{array}{c}\text { Surcote } \\
\text { Port Vendres } \\
\text { (Obs 18.3 ans) }\end{array}$ & $\begin{array}{c}\text { Surcote } \\
\text { Sète } \\
\text { (Obs 31.3 ans) }\end{array}$ & $\begin{array}{c}\text { Vague } \\
\text { Candhis Leucate } \\
\text { (Obs 7.7 ans) }\end{array}$ & $\begin{array}{c}\text { Vague ANEMOC } \\
\text { (Mod 30 ans) }\end{array}$ & $\begin{array}{c}\text { Vague GoLWA } \\
\text { (Mod 30 ans) }\end{array}$ \\
\hline 1 an & - & - & 4.07 & 3.74 & 3.33 \\
10 ans & 0.44 & 0.53 & 5.44 & 5.1 & 5.02 \\
50 ans & 0.56 & 0.63 & $6.83^{*}$ & 6.27 & 6.01 \\
100 ans & 0.61 & 0.68 & - & 6.84 & 6.4 \\
\hline
\end{tabular}

\subsubsection{Niveaux d'eau dans la lagune}

La lagune de Leucate communique avec le domaine marin par trois graus aménagés de faibles largeurs, le plus important étant localisé au fond du port de Port-Leucate. Ces graus, partiellement obstrués en période hivernale afin de bloquer la migration des poissons, rendent complexe le remplissage et l'évacuation de l'eau en cas de tempêtes (IFREMER, 2003). Les fortes pluies provoquent alors une aggravation de l'aléa côté lagune alors que la conjugaison des fortes houles et de la surcote entraine des débordements dans le port. Ces variations de niveau d'eau de part et d'autre du lido sont prises en compte dans les scénarios envisagés.

\subsection{Traitement des données topo-bathymétriques}

Un modèle numérique de terrain (MNT) terre/mer a été constitué à partir de données bathymétriques (mer, étang, chenaux portuaires) et de données topographiques (partie terrestre et bande littorale jusqu'à $10 \mathrm{~m}$ de profondeur) issus du produit Litto-3D (2011, source IGN).

La propagation de la submersion marine est conditionnée par (i) les éléments topographiques et structurants qui peuvent bloquer les écoulements, (ii) l'occupation du sol qui se traduit en termes d'état de surface du terrain et de "résistance à l'écoulement", (iii) les connexions hydrauliques pouvant faciliter ou bloquer le passage d'obstacles à 
Côtes méditerranéennes menacées :

Risques et défis dans le contexte du changement climatique

l'écoulement. Ces divers particularités géométriques et surfaciques ont été prises en compte via :

- les caractéristiques des surfaces bâties extraites du Modèle Numérique d'Elévation (MNE) brut (2011, source IGN) ;

- les éléments structurants (murets de front de mer) ou lignes de contraintes et leurs caractéristiques à partir de levés au GPS différentiel ;

- les principales connexions hydrauliques (buses, écluses, ponceaux) et leurs caractéristiques géométriques (hauteur, largeur de section, fonctionnement) ;

- un coefficient de rugosité en fonction du type de sol (broussailles, pelouse, béton, zone arborée, ...).

Les MNE issus de ces traitements sont utilisés pour constituer un emboitement de 2 grilles de calcul. La grille principale de résolution de l'ordre de 20 m couvre l'ensemble du système lagunaire. Une seconde grille de résolution 5 m (cf. Figure 1), permettant de représenter les principaux éléments urbains, couvre la commune de Leucate.

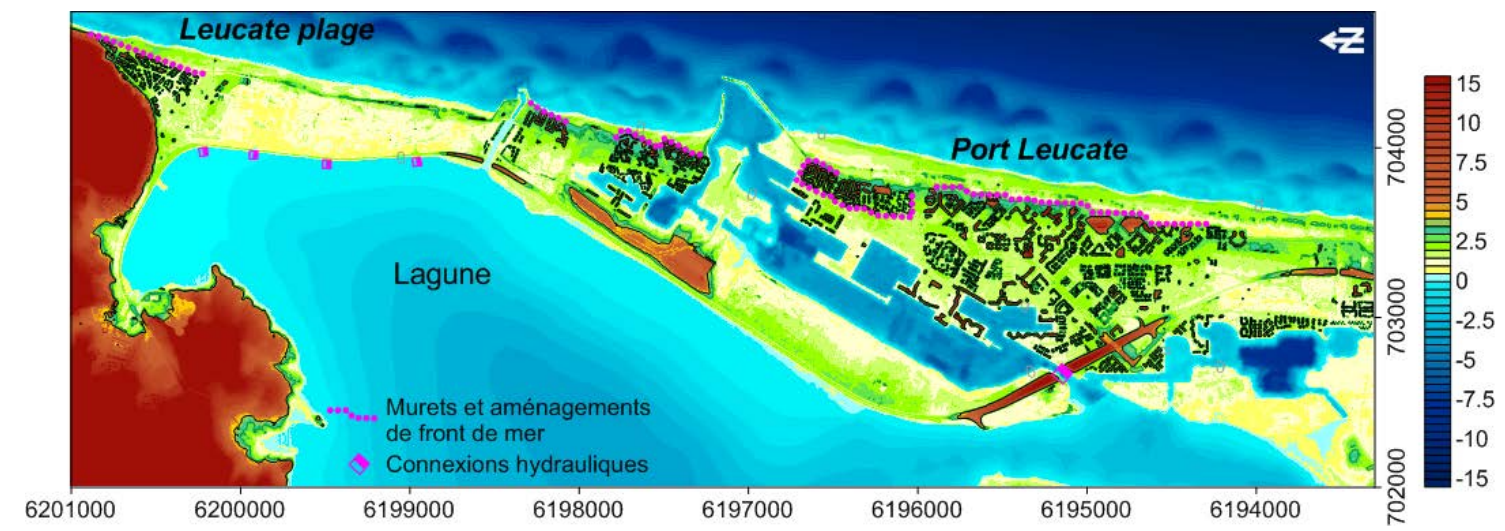

Figure 1. MNE traité du site d'étude (résolution $5 \mathrm{~m}$ ) et exploité pour la modélisation de submersions marines.

\subsection{Les modèles numériques}

La modélisation des niveaux d'eau à la côte a été réalisée à partir du modèle MARSSWAN. Ce couplage permet de simuler les variations des niveaux d'eau et les interactions avec les conditions de vague (très importantes dans le domaine proche côtier).

Le modèle MARS2DH (Licence IFREMER) permet de simuler les conditions hydrodynamiques associées : (i) en mer, aux variations du niveau d'eau provoquées par la marée, la pression atmosphérique et les vents, (ii) à terre, à la propagation des flux d'inondation pour des submersions par débordement ou suite à la défaillance d'ouvrage de protection côtière.

Le modèle SWAN permet de simuler la propagation des vagues du large à la côte en tenant compte des interactions des vagues entre elles et de la bathymétrie. Il détermine également la surélévation du niveau moyen (wave setup) lors du déferlement. 
Le couplage de ces deux modèles MARS-SWAN permet de simuler les variations des niveaux d'eau en tenant compte des interactions avec les conditions de vagues et permet ainsi d'intégrer l'ensemble des phénomènes susceptibles de contribuer à la surélévation du niveau d'eau à la côte lors des tempêtes.

Dans les secteurs où les submersions susceptibles de se produire sont de type franchissement par paquets de mer, l'estimation des volumes débordant est réalisée via des modélisations 1D avec le code SWASH. Ce modèle dit « vague à vague » est utilisé ici afin d'estimer les hauteurs atteintes par le jet de rive et les volumes d'eau susceptibles de franchir les ouvrages de front de mer en fonction de leur géométrie. Les volumes d'eau intégrés sur la longueur de la zone concernée sont réinjectés dans le modèle de submersions en arrière des ouvrages pour reproduire la propagation des volumes d'eau franchissant.

\section{Résultats attendus}

Deux évènements tempétueux récents ont été utilisés pour estimer la qualité des simulations numériques. Cette procédure de validation est basée sur la reproduction de deux évènements: la tempête d’octobre 2010 qui a généré des submersions par débordement et franchissement de paquets de mer localisés, à la fois côté mer et côté étang et la tempête de mars 2013 où la rupture d'un muret sur le front de mer de Leucate-plage a entraîné une submersion importante. Outre la validation des modèles, ces deux types de submersion serviront de base à l'élaboration de scénarios prospectifs. Dans le cadre d'une analyse prospective de l'aléa, des scénarios extrêmes seront élaborés, associant conditions de tempêtes les plus sévères (issues d'une analyse statistique des évènements passés) et défaillances sur des ouvrages de front de mer devant les quartiers les plus exposés. Une attention particulière sera portée à la pérennité des axes de communication, afin d'évaluer le temps nécessaire pour évacuer les quartiers les plus vulnérables. Enfin, des scénarios prenant en compte l'élévation du niveau marin seront modélisés, pour suivre l'évolution possible de l'aléa et anticiper les adaptations nécessaires des mesures de gestion des risques et des crises.

\section{Conclusions}

Le travail réalisé dans le cadre de projet CRISSIS consiste en une modélisation fine des processus marins et lagunaires à l'origine des submersions sur la commune de Leucate. Il envisage également la simulation réaliste des écoulements à terre par la bonne représentation des milieux urbains et des aménagements (murets, ouvrages hydrauliques...) pouvant constituer des freins ou provoquer l'aggravation de l'aléa. La reproduction d'évènements historiques récents et les scénarios réalisés ont vocation à offrir des supports de réflexion pour mieux connaitre l'aléa de submersion et tester via des exercices de crise, l’opérationnalité du Plan Communal de Sauvegarde adopté en 
Côtes méditerranéennes menacées :

Risques et défis dans le contexte du changement climatique

2013 par la commune. Ils serviront également à la délimitation de secteurs vulnérables où sera enquêtée la perception du risque par la population.

Remerciement : Des remerciements sont adressés au Conseil Supérieur à la Formation et Recherche Stratégique (CSFRS) et aux divers services de la Mairie de Leucate participant au projet.

\section{Références bibliographiques}

ANSELME B., DURAND P., THOMAS Y.F., NICOLAE-LERMA A. (2011). Storm extreme levels and coastal flood hazards. A parametric approach on the French coast of Languedoc (district of Leucate). Comptes Rendus Geosciences, Vol. 343(10), pp 677-690. http://dx.doi.org/10.1016/i.crte.2011.07.006

BALOUIN Y., BELON R. (2012). Evaluation des submersions marines lors des tempêtes en Languedoc-Roussillon. Atlas cartographique. Rapport final, BRGM/RP61306-FR, 124 p.

BALOUIN Y., BELON R., DELVALLEE E., LAMY M., BODERE G. (2011). ROL Etude complémentaire sur l'impact des tempêtes sur le littoral de la Plaine Orientale de Corse - Cartographie de la submersion marine et de la sensibilité à l'érosion côtière et de l'exposition des enjeux. BRGM/RP-59724-FR, 39 p.

CETMEF (2013). Analyse des surcotes extrêmes le long des côtes métropolitaines. $128 \mathrm{p}$.

IFREMER (2003). Réseau de suivi lagunaire du Languedoc-Roussillon. Bilan des résultats 2002. Rapport de la Direction de l'Environnement et de l'Aménagement littoral, laboratoire de Sète, 234 p.

LE ROY S., PEDREROS R., NICOLAE LERMA A. (2013). Modélisation de la submersion marine en milieu urbain. Rapport final. BRGM/RP-63477-FR, 84 p.

PEDREROS R., VINCHON C., LECACHEUX S., DELVALLÉE E., BALOUIN Y., GARCIN M., KRIEN Y., LE COZANNET G., POISSON B., THIEBOT J., BONNETON P., MARCHE F. (2011). Multi models approach to assess coastal exposure to marine inundation within a global change context. Poster, EGU (European Geosciences Union) General Assembly, Wien, 03-08/04/2011.

GARCIN M., NICOLAE LERMA A., PEDREROS R. PARIS F. (2012). Evaluation de la submersion marine maximale à l'échelle régionale. Rapport final, BRGM/RP-62259FR, p.97.

VINCHON C., BARON-YELLES N., BERTHELIER E., HÉRIVAUX C, LECACHEUX S., MEUR-FEREC C., PEDREROS R., REY-VALETTE H., RULLEAU B. (2010). MISEEVA : Set up of a transdisciplinary approach to assess vulnerability of the coastal zone to marine inundation at regional and local scale, within a global change context. Littoral 2010, London, sept 2010. 